\title{
AN ENSEMBLE-BASED CHANGE-POINT DETECTION METHOD FOR IDENTIFYING UNEXPECTED BEHAVIOUR OF RAILWAY TUNNEL INFRASTRUCTURES
}

\author{
Matteo Vagnoli ${ }^{*}$, Rasa Remenyte-Prescott ${ }^{1}$ \\ ${ }^{1}$ Resilience Engineering Research Group, the University of Nottingham \\ Science Road, University Park, \\ NG7 2RD, Nottingham, United Kingdom \\ *corresponding author: Matteo.vagnoli@nottingham.ac.uk
}

\begin{abstract}
A large amount of data is generated by Structural Health Monitoring (SHM) systems and, as a consequence, processing and interpreting this data can be difficult and time consuming. Particularly, if work activities such as maintenance or modernization are carried out on a bridge or tunnel infrastructure, a robust data analysis is needed, in order to accurately and quickly process the data and provide reliable information to decision makers. In this way the service disruption can be minimized and the safety of the asset and the workforce guaranteed. In this paper a data mining method for detecting critical behaviour of a railway tunnel is presented. The method starts with a pre-processing step that aims to remove the noise in the recorded data. A feature definition and selection step is then performed to identify the most critical area of the tunnel. An ensemble of change-point detection algorithms is proposed, in order to analyse the critical area of the tunnel and point out the time when unexpected behaviour occurs, as well as its duration and location. The work activities, which are carried out at the time of occurrence of the critical behaviour and have caused this behaviour, are finally identified from a database of the work schedule and used for the validation of the results. Using the proposed method, fast and reliable information about infrastructure condition is provided to decision makers.
\end{abstract}

Keywords: Structural Health Monitoring (SHM); Data mining; Change-point detection; Ensemble of change-point detection methods 


\section{Introduction}

The size of the European railway network is expected to continuously increase in order to transport most of the long-distance passengers and freight by 2030 [IRA, 2015]. Railways are, indeed, among the most emission-efficient transportation systems, and electric trains can offer a carbon-free journey (if they are powered using nuclear or renewable power sources). However, the European railway network is ageing, and its materials are degrading due to environmental threats (such as traffic, wind and temperature changes [Lee et al., 2005; Chattopadhyay and Reddy, 2007; Boller et al., 2015]. The railway infrastructure is thus calling for: $i$ ) a real-time condition monitoring of its health state over time; $i$ ) maintenance activities to restore the health state to a new safe condition; and iii) renewal activities to enhance the performance of the railway network, in terms of comfort to passengers, speed, safety and availability of the service [Baxter, 2015]. Structural Health Monitoring (SHM) strategies are needed to guarantee the safety, reliability and availability of the infrastructure both during normal operation of the railways and during maintenance and modernization activities [Lee et al., 2010; Chen and Wang, 2017]. Particularly, the former requires an SHM method in order to accurately monitor the infrastructure behaviour over time, by identifying ongoing degradation mechanisms of the materials. The latter requires an SHM strategy in order to monitor the infrastructure behaviour in the short-period, by ensuring the safety of the workforce and understanding whether the infrastructure behaviour during the work activities is within the predicted safety limits [Brownjohn, 2007; Rajabi et al., 2017].

SHM strategies are especially necessary for underground infrastructure during their maintenance and modernization activities. In fact, the uniqueness of each underground infrastructure can lead to unexpected behaviour of the in-field structure during the work activities [Bhalla et al., 2005]. For example, the UK railway network is subject to an electrification process, which aims to provide cleaner, quicker and more comfortable trains. The old infrastructure of the UK railway network does not usually have the clearance necessary to install the Overhead Line Equipment (OLE) system, which consists of $25 \mathrm{kV}$ AC live conducting wires, insulators and supporting equipment [Kilsby et al., 2017]. Hence, maintenance and modernization activities of railway infrastructures are planned, in order to provide the necessary clearance for the OLE system. In the case of tunnels, the way chosen to provide the necessary clearance for the OLE is: $i$ ) to remove the track, sleepers and ballast; $i$ ) to excavate into the sub-formation in order to obtain a new lowered ground; iii) to re-establish the ballast, sleepers and track to the new lower level that provides the necessary clearance for the OLE system. During these works, a real-time SHM system is required with the aim of monitoring the tunnel behaviour. The safety of the workforce is then guaranteed by verifying whether the predicted safety limits are respected by the actual behaviour of the tunnel [Brownjohn, 2007].

Several long-term SHM systems for tunnel infrastructure are presented in literature [Bhalla et al., 2005; Li et al., 2015; Rajabi et al., 2017; Wang et al., 2017], with the aim of developing and studying an optimal measurement system to monitor and predict the behaviour of the tunnel. For example, [Bhalla et al., 2005] present a comprehensive review of measurement strategies for a tunnel, by analysing and discussing the positive effect of the SHM on the tunnel 
life cycle. [Li et al., 2015] introduce a new wired sensor strategy to monitor the convergence of a tunnel under work activities; [Rajabi et al., 2017] discuss an Artificial Neural Network (ANN)-based method in order to predict horizontal displacements of a tunnel, by relying on the data provided by a 2D Finite Element Model (FEM) of the infrastructure; [Wang et al., 2017] present a long-term SHM system in order to assess the safety of a number of tunnels in China. A large amount of data is however generated by these SHM methods continuously. Data mining techniques are therefore required to analyse this data automatically, accurately and rapidly [Duan and Zhang, 2006]. Indeed, data mining techniques are able to transform the recorded data into valuable information for decision makers, by pointing out vulnerabilities of the tunnel. As a consequence, the safety of the asset and the workforce can be improved by taking rapid informed decision [Alves et al., 2015; Zhou et al., 2015; Li et al., 2016].

In this paper, an ensemble-based data mining method is proposed in order to detect the unexpected behaviour of a railway tunnel. The tunnel is subjected to enhancement works, which are necessary for the installation the OLE system during the electrification process of the UK railway network. An SHM system is installed on the tunnel for monitoring the displacements of the tunnel during the works. An FEM of the tunnel has been developed by the contractor of the works in order to predict the displacement of the tunnel during the works. The FEM showed that the expected displacement of the tunnel should remain around the value of zero during each phase of the works, and, if the tunnel would converge, the displacement would increase at a rate of $0.001 \mathrm{~mm} / \mathrm{h}$. A data mining analysis is proposed in order to analyse measurements over one month, and identify unexpected behaviour of the tunnel, i.e. the behaviour that does not agree with the FE predictions. The works that are carried out on the tunnel at the time of the unexpected behaviour are also investigated. The recorded data are firstly analysed by using a pre-processing step, which removes the noise of the data. Then, a feature definition and selection process is adopted in order to identify the critical locations of the tunnel, by the means of a K-means algorithm [Jain, 2010]. The unexpected behaviours, which are measured at the critical location on the tunnel, are evaluated by using a novel ensemble-based change-point detection method. The proposed method is used to identify the time when the tunnel starts to converge rapidly and point out the duration of the unexpected behaviour. The performance of four individual change-point detection algorithms is merged to detect and diagnose the most critical behaviour of the tunnel [Lavielle, 2005; Carslaw et al., 2006; Killick et al., 2012; Liu et al., 2013]. The works at the tunnel site that are carried out at that time are investigated, with the aim of pointing out the causes of the unexpected behaviour. The ensemble-based change-point detection method is needed in order to identify the most critical change-point of the monitored tunnel behaviour. Indeed, single change-point methods, such as Cumulative Sum (CUSUM)-based [Carslaw et al., 2006] or probability distributionsbased [Liu et al., 2013] methods, are able to detect only abrupt changes in the data, without pointing out the most severe changes. Furthermore, the longer the duration of the monitored behaviour of the system, the higher the number of the abrupt changes, which are identified by an individual change-point method. Thus, the most severe change in the data can be lost among all the change-points [Killick et al., 2012]. Individual change-point methods are also usually unable to identify the duration of the most critical system behaviour, as their objective is to point out the moment when the data deviates from the average behaviour [Maleki et al., 2016]. For these reasons, individual change point methods can lead to identifying the incorrect works 
that might have caused the unexpected behaviour of the tunnel. Conversely, the proposed ensemble-based of change-point methods is able to identify the most critical change in the data, by highlighting its start and end time. In this way, only the information regarding the most critical behaviour of the tunnel is provided to the decision maker. At the same time, the corresponding works at the worksite at the time of occurrence of the most critical behaviour are provided to the decision maker. The performance of the proposed ensemble-based method is compared with the results of each individual change-point algorithm. In this way, the superior ability of the ensemble-based change point method in identifying the most critical change-point is demonstrated.

The remaining of the paper is organized as follow: section 2 introduces the change-points algorithms and the proposed ensemble-based method; section 3 describes the tunnel case study and the SHM system and the results; conclusion and remarks are provided in section 4.

\section{The proposed ensemble-based change-point detection method}

Change-point detection methods are developed in order to detect changes of the monitored behaviour of a system efficiently and reliably [Tartakovsky et al., 2014]. Change-point methods aim to identify the exact moment when the monitored variable of the system starts to deviate abruptly from an equilibrium level. Change-point detection algorithms are adopted in several frameworks, such as SHM and prognosis of gas turbines [Lipowsky et al., 2010; Maleki et al., 2016], variation of air pollution concentration [Carslaw et al., 2006], variation of climate parameters in order to monitor climate change characteristics [Reeves et al., 2007], failure of pipes in chemical industries [Tickle et al., 2010]. However, change-point methods are usually unable to point out the most critical change-point clearly, i.e. the change-point where the monitored variable experiences the highest variation. In fact, the most critical change point is identified among all the change-points of the system. The duration of the unexpected changes is also not assessed. Furthermore, the choice of an individual change-point algorithm can jeopardize the reliability and robustness of the data analysis, due to different results of the individual change-point detection methods. For these reasons, in this paper we propose a novel ensemble-based change-point method to analyse a large database of displacements of a railway tunnel, by coupling the performance of four individual change-points methods. In this way, the proposed ensemble-based method is able to point out the most critical change-point of the system, providing its duration and possible causes. As a consequence, the reliability and robustness of the data mining analysis are expected to improve accordingly. Decision makers can then schedule future work activities by using the results of the ensemble-based changepoint method directly. Indeed, the most critical behaviour of the tunnel is pointed out clearly, in terms of both duration and corresponding works at the worksite.

In what follows, the theoretical background of the four individual change-point algorithms is presented briefly, and then the proposed ensemble-based method is introduced. 


\section{Change-point methods: theoretical background}

The change-point analysis can be divided in two groups - real-time and retrospective detection: the former aims to identify a change-point of system behaviour as soon as it occurs; the latter aims to identify a change-point of system behaviour by analysing the history of the monitored par:dmeter. The focus in this paper is on the retrospective change-point analysis, which provides more robust and accurate detection than the real-time analysis [Liu et al., 2013]. Particularly, the aim is to identify the most critical change-point of the system behaviour, by assessing its duration and diagnosing its possible causes. In order to achieve this aim, an ensemble-based change-point method is developed by coupling the performance of four of the most commonly adopted change-point algorithms: $i$ ) a change-point detection method that relies on a relative probability density ratio, which is computed by using the Relative unconstrained Least-Squares Importance Fitting (RuLSIF) [Liu et al., 2013]. The RuLSIF method has demonstrated to provide very good results in identifying change-points through the assessment of a relative probability density-ratio [Fuez et al., 2015]; ii) a Cumulative Sum (CUSUM) change-point detection algorithm [Carslaw et al., 2006]. The CUSUM is one of the most popular changepoint method that has been adopted in many different research framework, such as air pollution concentration [Carslaw et al., 2006], failures of computer networks [Montes De Oca et al., 2010], functionality of animal brain activity [Koepcke et al., 2016], failures of water distribution networks [Misiunas et al., 2006]; iii) a change-point detection method that relies on the identification of changes of the mean value of the monitored system behaviour, by defining a penalty cost function [Lavielle, 2005]; iv) a change-point detection method that relies on the identification of changes of the slope of the monitored system behaviour, by using a Pruned Exact Linear Time (PELT) method [Killick et al., 2012]. The change-point methods iii) and $i v$ ), which rely on the same theoretical basis, have been chosen due to their efficiency and low computational burdensome. Indeed, as the length of the monitored parameters increases, the number of possible change-points also increases, and thus an efficient and fast detection of change-point is needed [Harchaoui and Levy-Leduc, 2010; Killick and Eckley, 2014].

\subsubsection{Theoretical basis of the adopted change-point algorithms}

The comprehensive description of the theory of these methods is out of the scope of this paper, and an interested reader can find the details of the theoretical basis of these methods in the provided references. However, in what follows a simplified theoretical description of these methods is provided:

i) The RuLSIF change-point method. This method aims to detect a change-point by assessing the probability density ratio, $\frac{p(t)}{p(t+n)}$, of samples of the monitored system behaviour, $Y(t)$, between time $t$ and $t+n$, where $n$ is the size of a time window. The samples $Y(t)$ and $Y(t+n)$ are defined as the $k$ behaviour of the system within the time window $n$, such as the displacements of the tunnel that are recorded during $k$ times 
consecutively. For example, Fig. 1 shows the monitored behaviour of the system such as the displacement of a tunnel, $y(t)$, which is collected at each time $t$ : its samples, $Y(t)$ and $Y(t+n)$, are the $k=3$ displacements of the tunnel recorded during a time window of size $n=5$, i.e. $Y(t)$ is defined by the measurements recorded at time $a, b$ and $c$, whereas $Y(t+n)$ is equal to the displacements at time $f, g$ and $h$. The ratio of the probability densities, $\frac{p(t)}{p(t+n)}$, between $Y(t)$ and $Y(t+n)$ can be then assessed by using the RuLSIF strategy.

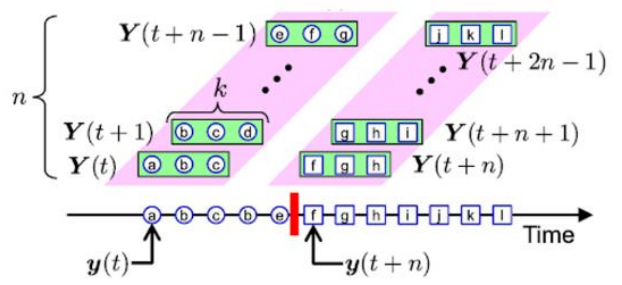

Fig. 1. Graphical relationship between the monitored behaviour of the system, $y(t)$, and its samples, Y(t) [Liu et al., 2013].

Particularly, the change-point is estimated by monitoring the evolution over time of an $\alpha$-relative Pearson (PE) divergence index, which depends on the Gaussian kernel estimation of the density-ratio:

$$
P E \propto \sum_{i=1}^{n} \theta_{i} K\left(Y, Y_{i}\right)
$$

where the Gaussian kernel function, $K$, is computed as:

$$
K\left(Y, Y^{\prime}\right)=\exp \left(-\frac{\left\|Y-Y_{i}\right\|^{2}}{2 \sigma^{2}}\right)
$$

$\sigma$ in Eq. (2) is the width of the kernel.

The parameters, $\theta_{i}$, are to be found from the data [Liu et al., 2013]. Eq. (1) and Eq. (2) show that the PE index increases as the distance between the samples $Y$ and $Y_{i}$ increases, i.e. the probability that a change-point occurred increases as the difference between the two samples of the monitored system behaviour increases.

ii) The CUSUM change-point detection algorithm. Given a time series of monitored behaviour of the system, such as the displacement of a tunnel at consecutive times $1,2, \ldots, m, y(t)=\left[\mathrm{y}_{1}, \mathrm{y}_{2}, \ldots, \mathrm{y}_{m}\right]$, where $m$ is the size of the measured behaviour of the system, the CUSUM chart is developed by assessing the cumulated difference between each value $y_{i}$ of $y(t)$ and the mean value of the displacement pattern, $\bar{y}$ : 


$$
S_{i}=S_{i-1}+\left(y_{i}-\bar{y}\right) \text { for } \mathrm{i}=1,2, \ldots, m
$$

Eq. (3) shows that when the system behaviour is measured, the cumulative difference, $S_{i}$, is computed as the difference between the mean value of the measured behaviour of the system, $\bar{y}$, (e.g. the mean value of the recorded displacement of a tunnel) and the value of the system behaviour at each time, $y_{i}$, (e.g. the displacement of the tunnel at each time step).

The difference between the maximum value of Eq. (3), $S_{\max }$, and the minimum cumulated difference, $S_{\min }$, is the maximum variation of the CUSUM, $\Delta S_{\max } . \Delta S_{\max }$ is used in order to evaluate if a change of the monitored behaviour of the system has occurred. Indeed, once $\Delta S_{\max }$ for the original recorded data is computed, the values of the monitored behaviour of the system, $y(t)$, are randomly resampled for 1000 times, and the CUSUM process is repeated for each resampled trial. As proposed by [Carslaw et al., 2006], we consider that a change has occurred on the recorded data $y(t)$ when the $\Delta S_{\max }$ of $y(t)$ is higher than the $\Delta S \max$ of the $95 \%$ of the randomly resampled trials. Therefore, a change point is estimated to have occurred at the time of occurrence of the maximum CUSUM value, $\triangle S \max$, if a $95 \%$ confidence level is achieved. Finally, multiple change-points can be detected by dividing the monitored behaviour data $y(t)$ in two parts, one for each side of the identified change-point, and repeating the CUSUM analysis for each part. The CUSUM analysis is terminated when no change-point is detected in each analysed part of $y(t)$.

iii) The penalty cost function-based change-point detection method. The existence of change-points of the monitored behaviour of the system is investigated by minimising an objective cost function, $C$. Particularly, assume that the monitored behaviour, $y(t)$, shows $l$ change-points, which occur at time $\tau_{1: l}=\left(\tau_{1}, \tau_{2}, \ldots, \tau_{l}\right)$. The monitored behaviour, $y(t)$, is divided in $l+1$ segments, $y_{\left(\tau_{j-1}+1\right): \tau_{j}}$. The cost function for each segment, which needs to be minimized, can be defined as follows:

$$
\sum_{j=1}^{l+1}\left[C\left(y_{\left(\tau_{j-1}+1\right): \tau_{j}}\right)\right]+\beta f(l)
$$

where $\beta f(l)$ is a penalty function that is usually linear with the number of the change-points, $\beta f(l)=\beta \cdot l$ [Killick et al., 2012]. The cost function $C$ is defined by using features of the monitored system behaviour. For example, the mean value, the root mean square, the standard deviation, etc. of the system behaviour can be used as features that define the objective of the cost function $C$. A change-point is identified at time when the cost function is minimized, i.e. at time when the chosen feature of the system behaviour changes suddenly. In Section 3.4 a cost function that aims to detect change-points by looking for changes of the mean value of the system behaviour is presented. The penalty parameter $\beta$ is optimized by using an 
iterative procedure with the aim of minimizing the cost function, $C$ [Lavielle, $2005]$. However, the optimal choice of the penalty function, $\beta f(l)$, and the penalty parameter, $\beta$, depend on several parameters that are unknown a-priori, such as the length of the data and number of change-points [Killick and Eckley, 2014].

iv) The PELT-based change-point detection method. This method identifies the change-points of the monitored behaviour of the system, $y(t)$, by relying on the same theoretical approach described for method iii), i.e. by minimizing the cost function, $C$. However, a pruning strategy is applied in order to improve the computational speed and efficiency of the change-point search. Hence, the values of the monitored behaviour of the system, $y(t)$, that cannot minimize the cost function, $C$, are removed from the analysis. In Section 3.4 a cost function that aims to detect changepoints by identifying changes on the slope of the system behaviour by considering an autoregressive linear model for the individual change-point method is presented.

\section{The proposed ensemble-based change-point detection method}

2.2

The individual change-point methods described in section 2.1.1, are able to identify efficiently abrupt change-points of the monitored variable of the system. However, the most critical change-point, which occurs when the monitored variable experiences the highest variation, is identified among all the change-points, experienced by the system. The performance of each individual change-point method is different, and consequently the reliability and robustness of the change-point detection analysis is influenced by the choice of a change-point method. The duration of the change of the system is also not assessed by these individual methods. The four individual change-point detection algorithms are then merged together in an ensemble strategy, in order to point out the most critical change-point of the monitored behaviour of the system, by identifying its duration. The following criteria for identifying the start and end of the changepoint are proposed Eq. (5-6) and (7-8), respectively:

$$
\text { 1. } \max \left\{\left|\frac{\delta y_{1: \tau_{1}}\left(\tau_{P}\right)}{\delta t}\right|\right\}
$$

where $\tau_{P}$ is defined as follows:

$\tau_{P}=\left\{\min \left(\tau_{1}^{k}, \tau_{1}^{q}\right)+\Delta|| \tau_{1}^{k}-\tau_{1}^{q} \mid \leq \Omega\right\}$, with $k=1, \ldots, M-1 ; q=k+1, \ldots, M$

2. $\min \left\{\left|\frac{\delta y_{\tau_{l-1}+1: \tau_{l}}\left(\tau_{P^{*}}\right)}{\delta t}\right|\right\}$

where $\tau_{P^{*}}$ is defined as follows: 


$$
\tau_{P^{*}}=\min \left(\frac{1}{N} \sum_{n^{*}=1}^{N} t_{n^{*}}+\Phi, \max \left(\tau_{l}^{k}, \tau_{l}^{q}\right)+\Phi|| \tau_{l}^{k}-\tau_{l}^{q} \mid \leq \Omega\right), \text { with } k=1, \ldots, M-1 ; k^{*}=k+1, \ldots, M
$$

where $M$ is the number of the individual change-point algorithms adopted, i.e. four in this example; $N$ in Eq. 8 is the length of the interval, where $P E \geq \Theta ; \tau_{1}^{k, q}$ and $\tau_{l}^{k, q}$ represent the first and $l$-th change-point identified by each individual change-point algorithm, respectively. Finally, $\Theta, \Omega$ and $\Delta$ are constant that need to be defined by the user. $\Theta$ is a threshold for the PE coefficient that needs to be chosen in order to emphasise the detection ability of the most critical change-point in the behaviour of the system, by neglecting other small changes [Liu et al., 2013]. The constants $\Omega$ and $\Delta$ need to be defined by the user when considering the nature of the case study. For example, if the ensemble-based method is applied for monitoring the existence of change in behaviour in a computer network, $\Omega$ and $\Delta$ need to be as low as possible, i.e. one second or less. Indeed, a computer network system manages large amount of information continuously, at each second or a fraction of a second. On the contrary, if the ensemble-based method is to be applied to monitor possible changes of the behaviour of a civil infrastructure, such as a bridge or tunnel, $\Omega$ and $\Delta$ will be set to larger values. In fact, the SHM measurement system can provide a measurement of the infrastructure behaviour every hour. As a result, $\Omega$ and $\Delta$ require to be optimized by considering the nature of the case study, and using expert knowledge elicitation.

Eq. (5) shows that the ensemble-based strategy is able to identify the initial change-point of the most critical unexpected behaviour of the system, by looking for the maximum variation of the monitored system behaviour at time $\tau_{P}$ (Eq. (6)). $\tau_{P}$ is the first change-point that is identified by at least two different individual change-point methods within $\Omega$ time of each-other, plus a constant $\Delta$.

Eq. (7) shows that the final point of the most critical unexpected behaviour of the system, i.e. the point where the critical unexpected behaviour ends, is identified by looking for time $\tau_{P^{*}}$. The point, $\tau_{P^{*}}$, in Eq. (8), is identified by looking for the minimum value between: $i$ ) the mean value of the time interval, where the PE coefficient is higher than the threshold $\Theta$; ii) the time where a change-point is identified by at least two different individual change-point methods within $\Omega$ time of each other, plus a constant $\Phi$. Again, $\Omega$ and $\Phi$ depend on the nature of the system analysed, and they are optimized considering the nature of the system under analysis. The ensemble-based method merges the performance of each individual change-point method in order to improve the reliability of the data analysis process. At the same time, the ensemblebased method is able to detect and diagnose the most critical change-point, by providing a robust and reliable analysis. Each individual change-point method identifies different changepoints of the monitored behaviour of the system, and so a reliable and robust analysis of the system behaviour can be influenced by the choice of the change-point algorithm. On the contrary, the proposed ensemble-based change-point method is able to provide the most critical change of the system behaviour, by coupling the results of the individual change-points methods. Therefore, the reliability and robustness of the identified change-point is improved. In what follows, the performance of the individual methods is compared with the performance 
of the ensemble-based strategy, in order to demonstrate the more robust and reliable results of the proposed strategy.

\section{A case study: data mining technique applied to a railway tunnel}

The proposed ensemble-based change-point method is applied to a database of real-time recorded displacements of a railway tunnel, which is subjected to electrification works. Particularly, during the electrification works, the track and the ballast of the rail are removed in order to excavate the sub-formation towards a new lowered level of the track, which provides the necessary clearance for the OLE system. However, when the track and the ballast are removed, the tunnel can start to converge due to changes of its support. A real-time SHM system is thus needed with the aim of monitoring the behaviour of the tunnel. A large amount of data is available from the SHM measurement system. Hence, a robust and rapid analysis of the recorded displacements of the tunnel is needed, in order to eventually identify unexpected behaviour of the tunnel. With this aim, a data mining procedure is proposed aiming to detect unexpected displacements of the tunnel during the works, by pointing out the time duration and the work activity at the tunnel at the moment of the unexpected behaviour. After a data preprocessing process that aims to delete the noise of the measurements, a feature definition and selection process is carried out, in order to identify, by the means of a K-means clustering algorithm, the locations where unexpected tunnel behaviour is measured. The critical behaviour is analysed by the means of the proposed ensemble-based change-point detection method, with the aim of identifying the duration of the unexpected variation of the tunnel displacements. The work activities that are carried out at the tunnel site at that moment are also provided.

Finally, it is worth noting that the proposed ensemble-based method is a post-processing data mining approach, which is able to identify and diagnose unexpected critical behaviour of an infrastructure, by analysing vast database of recorded data. At the same time, the identification of the works, which are carried out at the time of the unexpected critical behaviour, can suggest possible measures to mitigate the unexpected behaviour of the tunnel. Therefore, the proposed method can be used to address the general objectives of a risk analysis for a tunnel structure [Eskesen et al., 2004]. However, a comprehensive risk analysis for a tunnel structure is out of the scope of this paper. Methods such as fault tree analysis [Hyun et al., 2015], event tree analysis [Hong et al., 2009], Bayesian Network approach [Sousa and Einstein, 2012], Monte Carlo-based method [Rezaie et al., 2007] and fuzzy logic approach [Yazdani-Chamzini, 2014], which are proposed in literature, would need to be adopted in order to develop a comprehensive risk analysis for a tunnel structure.

\section{Introduction to the tunnel electrification works and the SHM system}

The electrification process of the UK railway network aims to develop a cheaper and cleaner railway system. For example, a reduction of maintenance activities is expected due to less wear of the railway track caused by electric trains, which are lighter than diesel trains, and also carbon-free journeys are provided by electric trains [Baxter, 2015]. One of the biggest 
challenges of the electrification process is the installation of the OLE system on aging railway lines. Figure 2(a) shows a tunnel where the OLE system cannot be installed. The following three main activities are scheduled in order to install the OLE: $i$ ) to remove the track, sleepers and ballast; $i$ ) to excavate into the sub-formation in order to obtain a new lowered ground; iii) to re-establish the ballast, sleepers and track to the new lower level that provides the necessary clearance for the OLE system. The works are carried out at intervals between 20 to 100 meters on the approach of the tunnel and inside the tunnel, in order to avoid a sudden sharp step in the track level. During these works, a real-time monitoring system is needed in order to continuously monitor the behaviour of the tunnel, by comparing the real convergence movements of the tunnel with those predicted by an FE model [Ordoñez et al., 2016]. The convergence of the tunnel can be measured by the means of optical and mechanical measurement systems: the former can rely on total stations and laser scan systems [Miura et al., 2005; Lato and Diederichs, 2014], whereas the latter can use distometer [Simeoni and Zanei, 2009] or fiber optical sensors [Mohamad et al., 2012]. However, the electrification works are carried out by working on two sets of track at different times. For example, whilst the track on the right hand-side of the tunnel is lowered, the track on the left hand-side needs to be accessible by trains in order to remove the ballast and move work materials. A measurement system that requires line-of sight within the tunnel or across the tunnel, such as laser distance measurement or total station, cannot be adopted. For these reasons, Shape Accel Array (SAA) sensors [Abdoun et al., 2009] are chosen by the contractor of the monitoring, which was appointed to study and install a monitoring system of the tunnel. Ten SAA sensors are installed within the first 100 meters of the tunnel, spaced at regular intervals along the tunnel length, in order to monitor a critical area of the tunnel where a void behind a section of the tunnel wall is discovered. Each SAA sensor is made of 23 orthogonally aligned accelerometers. Particularly, the sensors provide the relative displacement of the tunnel with respect to a reference point that measures zero always and has been numbered as sensor zero, as shown in Fig. 2(b). The frequency of the sensors is changed based on the type of the works that are carried out on the site, i.e. when the electrification works are carried out within the tunnel, the frequency is higher than when the works are carried out on the approaching of the tunnel. However, in this case-study a measurement of the displacement of the tunnel is provided for each hour, and, as a result, the frequency of the SAA is constant. An example of SAA sensor, which is composed of 23 accelerometers installed on the control points of the tunnel perimeter, is sketched in Figure 2(b), where the dotted line represents the SAA sensor. Finally, it should be noted that the available database, which is analysed by the proposed method, consists of the hourly measurements of the 230 sensors over a real-time monitoring period of 40 days. 


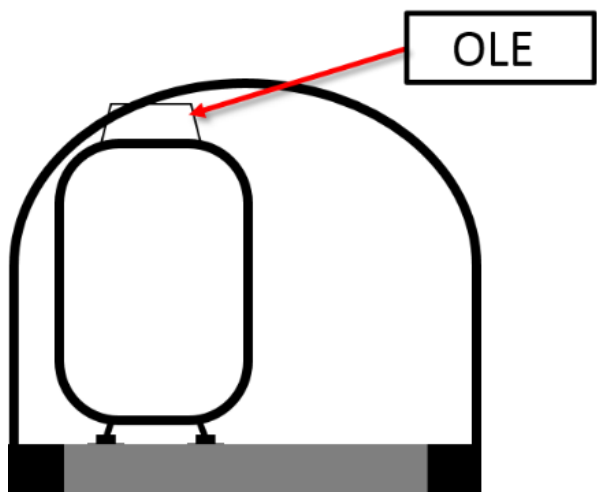

(a)

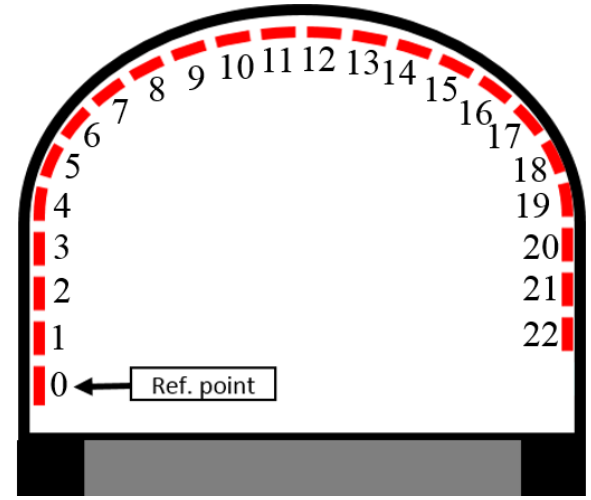

(b)

Figure 2. Example of clearance problem (a), and SAA monitoring system (b).

\title{
Step 1 - Pre-processing of the displacements of the tunnel
}

\begin{abstract}
3.2
A pre-processing of the measured displacements is needed in order to correct an off-set value problem of the sensors, which can occur during the monitoring period. The off-set problem can be caused by accidental knocks of the sensor during the works, and as a result, it needs to be corrected. The pre-processing is carried out by assessing the difference between two consecutive measurements of the tunnel displacements, which are recorded by the same sensor. Indeed, the analysis of the expected tunnel behaviour, which has been carried out by the experts of the works contractor by using an FE model, showed that the expected displacement of the tunnel should remain around the value of zero during each phase of the works. However, if the tunnel would converge, the displacement would increase at a rate of $0.001 \mathrm{~mm} / \mathrm{h}$. The experts suggested that if the tunnel displacement increases in one hour more than $0.3 \mathrm{~mm}$, i.e. the difference between two consecutive measurements of the tunnel displacement provided by the same sensor is higher than $0.3 \mathrm{~mm}$, an off-set problem of the sensor has occurred. The measurements need to be corrected by restoring the off-set of the previous hours accordingly. For example, Fig. 3(a) shows the raw data provided by a sensor with a wrong off-set value at time $578 \mathrm{~h}$, where the displacement of the tunnel jumps from $-0.56 \mathrm{~mm}$ to $16.14 \mathrm{~mm}$ in one hour. This behaviour is extremely unlikely to be caused by a real movement of the tunnel, and, more likely, it is caused by a knock of the sensor during the works. The off-set problem of the displacements leads to an incorrect assessment of the health condition of the tunnel. A preprocessing procedure is needed in order to re-establish the correct value of the displacement of the tunnel. Fig. 3(b) depicts the processed displacement, where the wrong off-set at time 578h is removed by adding an off-set of $-15.58 \mathrm{~mm}\left(\mathrm{~d}_{577}-\mathrm{d}_{578}=-15.58 \mathrm{~mm}\right)$ at time $578 \mathrm{~h}$. A preprocessing analysis is carried out on the displacements of the whole database, in order to correct the off-set problem, and consequently analyse the correct behaviour of the tunnel. Similarly, Fig. 3(c) shows an off-set error where the relative displacement drop at time 588h from $0.04 \mathrm{~mm}$ to $-2.6 \mathrm{~mm}$. After the pre-processing of the data the correct displacement of the tunnel can be analysed, as shown in Fig. 3(d).
\end{abstract}




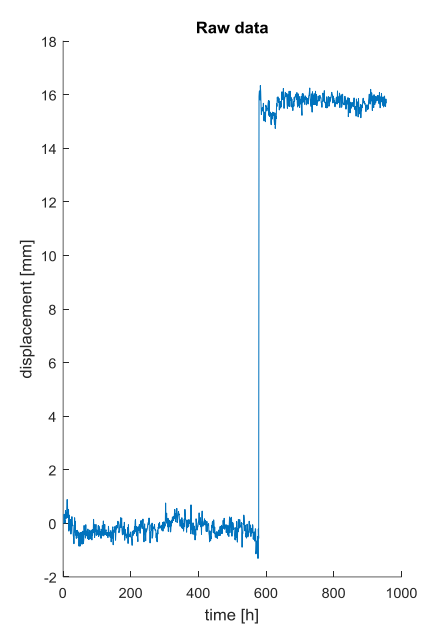

(a)

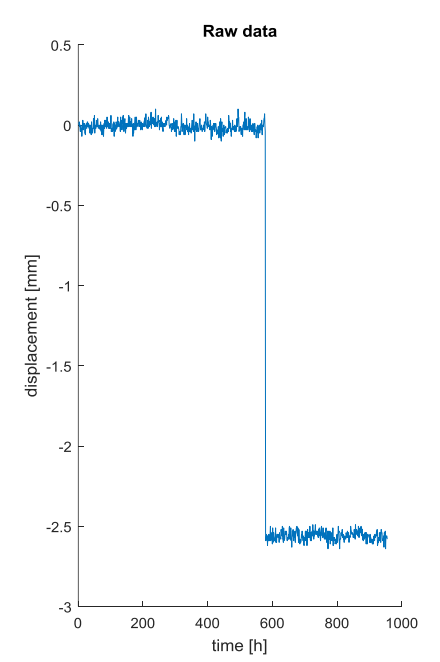

(c)

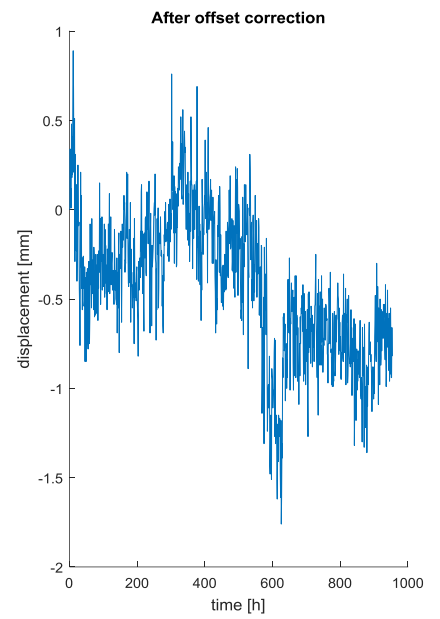

(b)

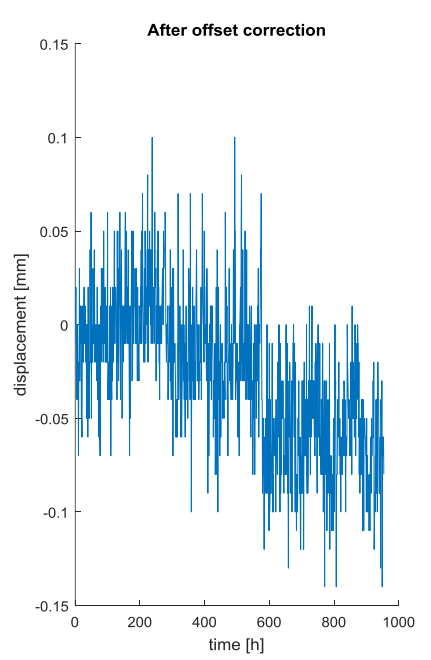

(d)

Figure 3. Displacements before (a-c) and after the off-set correction (b-d).

\section{Step 2 - Identification of critical location of the tunnel}

In order to point out the critical locations of the tunnel, which need to be analysed by the means of the proposed ensemble-based change-point detection method, a feature definition and selection process is developed, as shown in Fig. 4. In this way, the critical locations can be identified by selecting the features that optimize the performance of a K-means clustering algorithm. The displacements of each SAA in the tunnel are used as an input to a feature definition problem, where 11 statistical features (such as mean value, standard deviation, peak value, minimum, kurtosis, skewness, root mean square, median, interquartile range, $5 \%$ and 95\% percentile) of the displacements of the tunnel are evaluated. The 11 statistical features of the displacement of each SAA sensor are then used as an input to an iterative process, which aims to optimize the performance of a K-means clustering in terms of the compactness and separation of the clusters. The iterative process aims to maximize the silhouette index [Rousseeuw, 1987] of the tunnel behaviour belonging to each cluster, by grouping similar behaviour of the tunnel in the same cluster (compactness), and dissimilar behaviour in different 
clusters (separation). A group of candidate optimal features is selected by a Genetic Algorithm (GA) engine [Baraldi et al., 2016; Di Maio et al., 2016], and then it is used as an input to the $\mathrm{K}$-means algorithm, where the features are grouped by evaluating the different number of clusters (the number of cluster is assumed to be between 2 and 5). The performance of the clustering algorithm is evaluated by assessing the silhouette index of the clusters, i.e. for each behaviour of a cluster, the silhouette index is computed by assessing its similarity with the other behaviours of the same cluster (compactness) compared to those of other clusters (separation). The iterative process is repeated until the silhouette index is maximized, and thus, the optimal features and the clusters are identified.

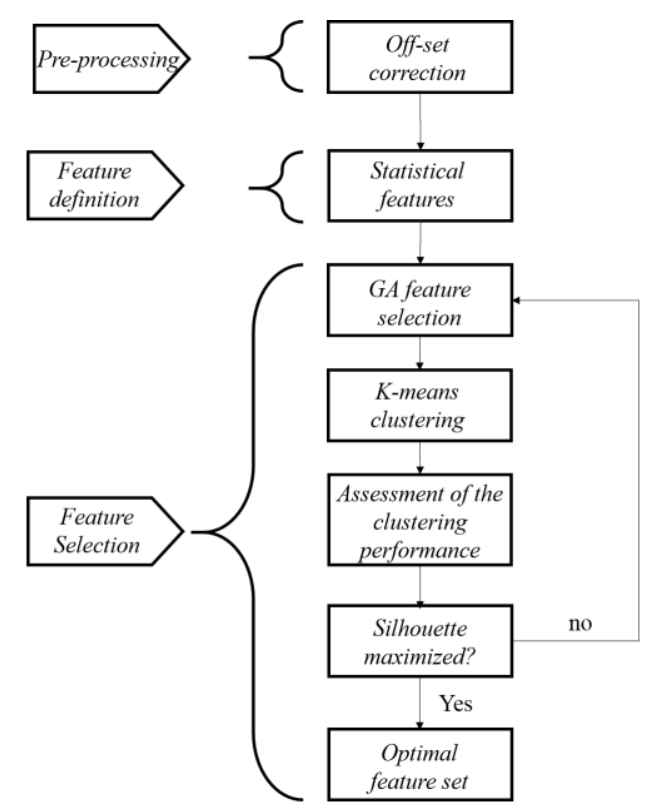

Figure 4. Feature definition and selection process.

7 of the 10 SAAs, which are installed along the tunnel, show displacements that are around the value of zero for most of the time of the observation, or slow increase over time of the works by respecting the prediction of the FE model. For that reason, these SAAs are excluded from further analysis. On the contrary, 3 SAAs show unexpected behaviour of the tunnel, and they need to be thoroughly analysed by the means of the proposed change-point method. In this way, we can investigate when and why the tunnel started to depart from the predicted displacement. The three critical SAAs are installed at 30, 40, and 80 meters inside the tunnel, respectively. Fig. 5 and 6 show the optimal features and clusters for the SAAs 30 and 80, by highlighting that the optimal number of clusters for each critical SAA is 5. The optimal set of features is different for each critical SAAs, i.e. the different behaviour of the tunnel, which is recorded by different SAA sensors, is clustered optimally by different statistical features.

\subsubsection{SAA installed 30m inside the tunnel (SAA30)}

Fig. 5(a) shows that the standard deviation, the peak value and the median are the best features in order to cluster the measurements of the tunnel recorded by the SAA installed $30 \mathrm{~m}$ inside the tunnel. The most critical clusters are those with the highest values of these features, i.e. 
cluster 1, 3 and 4, which are represented by circles, points and pointing-up triangles respectively in Fig. 5(a). Accordingly, the measurements of the tunnel, which have the highest variability, the maximum value of displacement and median, are those belonging to the most critical clusters, as shown in Fig. 5(b), where the displacements of clusters 1, 3 and 4 show an unexpected fast increase at the beginning of the works. The displacements of the tunnel, Fig. 5 (b), are monitored and reported in this paper in order to verify the results of the K-means clustering in identifying the most critical sensors of the tunnel, which are further analysed in Step 3. Clusters 1, 3 and 4 (and the related sensors, as shown in Fig. 5(b)) require to be analysed with the proposed ensemble-based change-point detection method, in order to detect the exact point when the tunnel started to converge rapidly. The duration of this unexpected behaviour and the works that are carried out at that time also need to be identified.
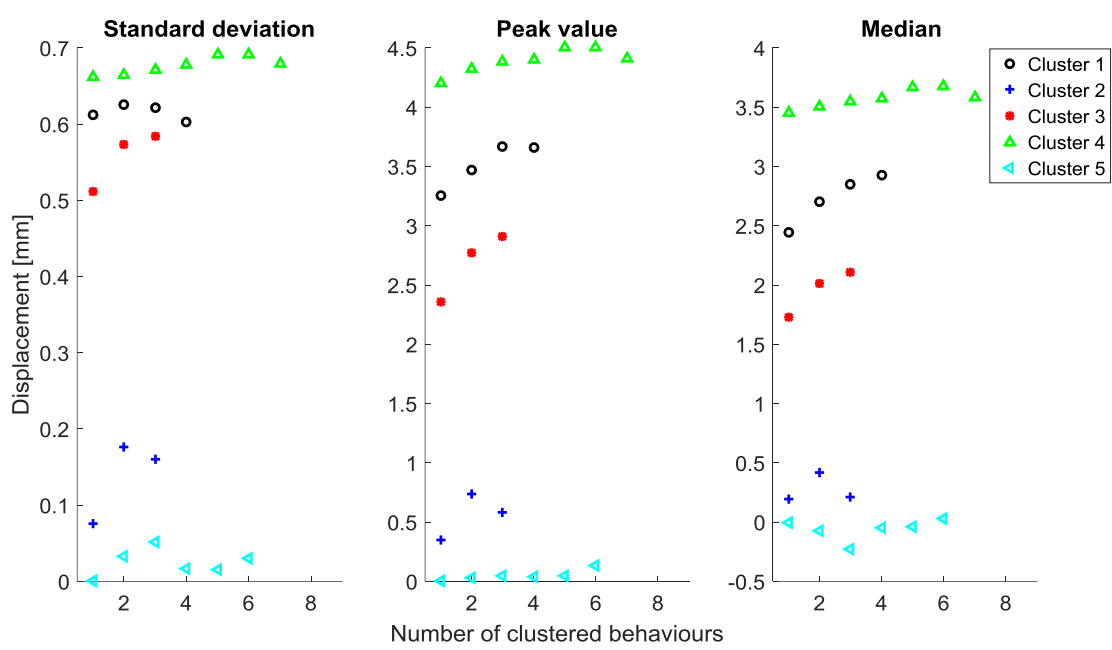

(a)
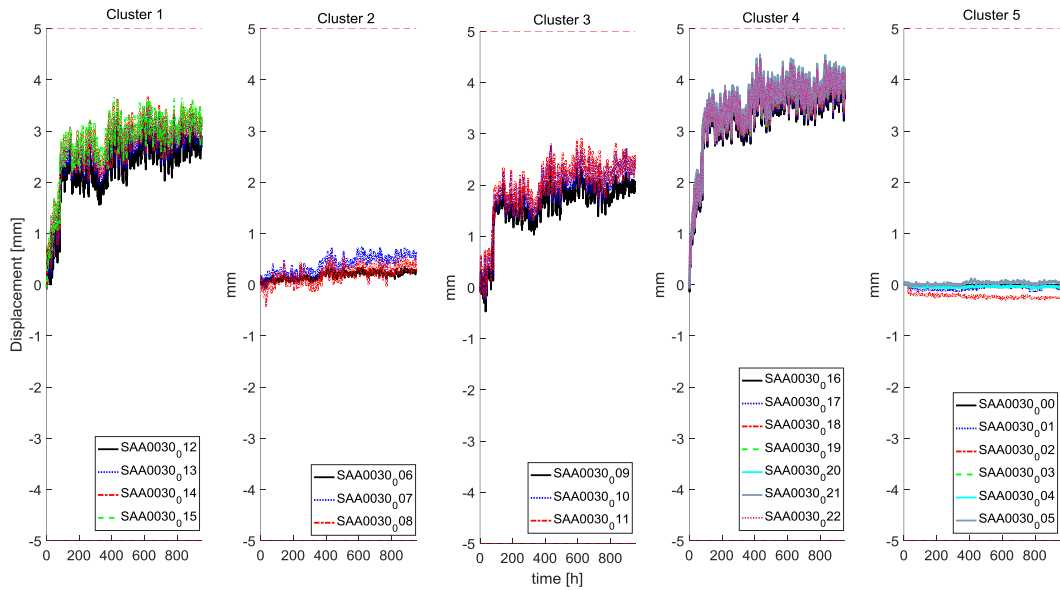

(b)

Figure 5. Optimal features (a) and grouped behaviours of the tunnel (b) measured by the SAA installed $30 \mathrm{~m}$ inside the tunnel.

\subsubsection{SAA installed 80m inside the tunnel (SAA80)}

Unexpected behaviours of the tunnel are also measured by the SAA installed at 80 meters inside the tunnel. For this SAA, the optimal features that allow to obtain compact and well separated 
clusters are the mean value, the root mean square and the median of the displacements. Therefore, the most critical clusters are those with the extreme values of these features, as shown by circles, points, pointing-up and pointing-backwards triangles in Fig. 6(a) for clusters 1, 3, 4 and 5, respectively. The tunnel behaviours that belong to these four clusters show an increase of the convergence of the tunnel at the beginning of the works, and consequently the analysis with the ensemble-based change-point detection method of these behaviours is needed.
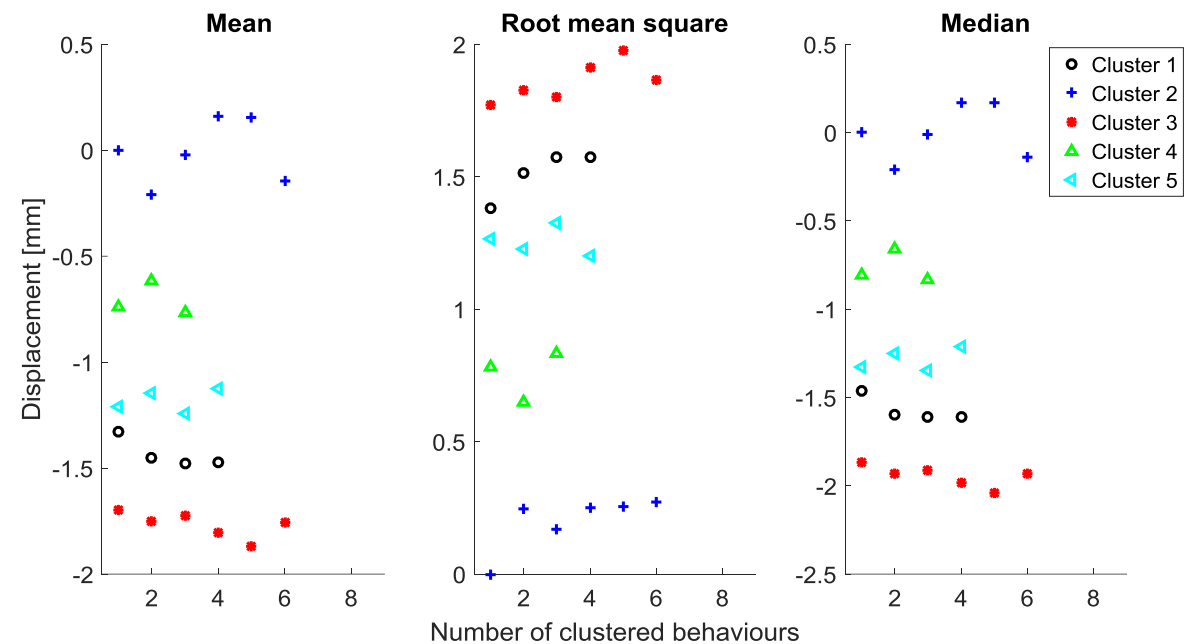

(a)
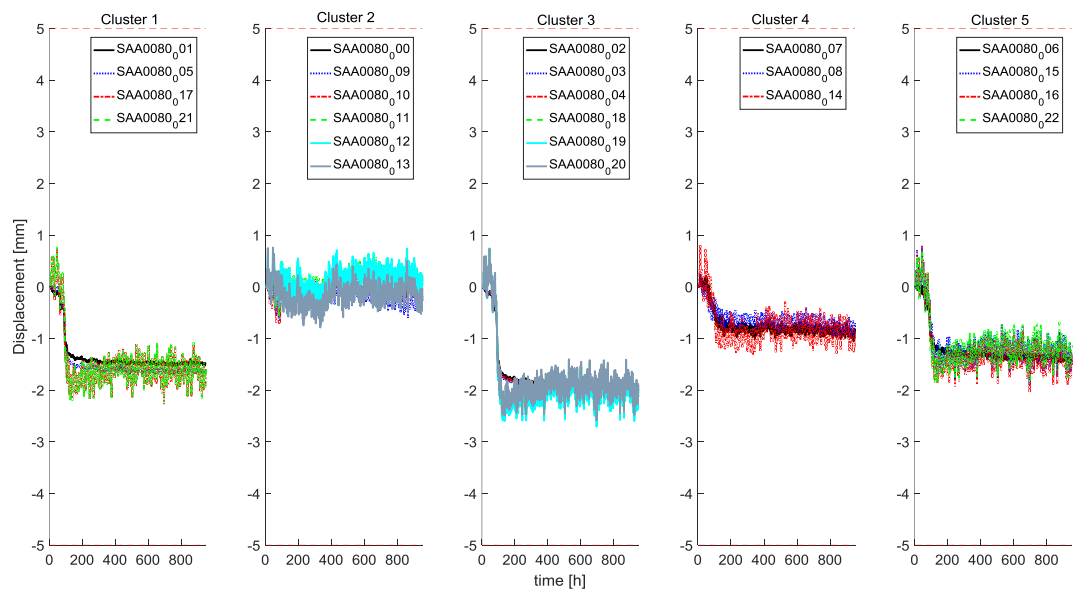

(b)

Figure 6. Optimal features (a) and clustered behaviours of the tunnel (b) measured by the SAA installed $80 \mathrm{~m}$ inside the tunnel.

\subsubsection{Discussion about the identification of critical SAAs of the tunnel}

The critical locations of the tunnel, i.e. those SAA sensors that have measured unexpected displacement of the tunnel, are identified by developing the feature definition and selection problem. The identified critical behaviour is further analysed in order to identify the duration of the unexpected critical behaviour, and the kind of works that are carried out at the worksite at that time. 
The characteristics of the identified locations of the tunnel are summarized in Table 1, where the optimal features, the number of critical clusters and sensors, and the location of the critical sensor in the SAA are described.

\begin{tabular}{|c|c|c|c|c|}
\hline SAA location [meter] & Optimal features & $\begin{array}{c}\text { Number of critical } \\
\text { clusters }\end{array}$ & $\begin{array}{c}\text { Number of critical } \\
\text { sensors }\end{array}$ & $\begin{array}{c}\text { Numbering label } \\
\text { of the critical } \\
\text { sensors }\end{array}$ \\
\hline 30 & $\begin{array}{c}\text { Standard deviation } \\
\text { Peak value } \\
\text { Median }\end{array}$ & 3 & 14 & From 9 to 22 \\
\hline 40 & $\begin{array}{c}\text { Mean value } \\
\text { Root mean square } \\
\text { Median }\end{array}$ & 3 & 13 & $\begin{array}{c}\text { From } 7 \text { to } 13 \text { and } \\
\text { from } 16 \text { to } 20\end{array}$ \\
\hline 80 & $\begin{array}{c}\text { Mean value } \\
\text { Root mean square } \\
\text { Median }\end{array}$ & 4 & 16 & $\begin{array}{c}\text { From } 1 \text { to } 8 \text { and } \\
\text { from } 14 \text { to } 22\end{array}$ \\
\hline
\end{tabular}

Table 1. Analysis of the critical SAAs.

The analysis of the critical clusters of the three SAAs shows that the sensors that have high numbering label (from 9 to 22), i.e. those on the right hand-side of the tunnel, as shown in Fig. 2(b), are those with the higher value of displacement, as shown in Table 1. However, the SAA installed at $80 \mathrm{~m}$ inside the tunnel, Fig. 6(b), shows high values of displacement on both sides of the tunnel, and as a result the infrastructure of the tunnel might have unknown critical issues at this point. This common behaviour can mean that the works are carried out on the right-hand side of the tunnel, and as a result the displacement of the tunnel on the right hand-side is higher than on the left-hand side, due to the temporary lack of the track and the excavation process. However, such detail information is not available on the database of the work activities, as only the information about the main work activity (e.g. excavation of the zone between 80 and 100 meters) is available.

In what follows, the behaviour of the critical clusters, which are identified by using the feature definition and selection process, are analysed further, in order to point out the time of occurrence, the duration and the possible causes of the unexpected fast convergence of the tâ.Anel.

\section{Step 3 - Change-point identification using the proposed ensemble-based methodology}

The measurements that have been identified to describe the critical behaviour of the tunnel are further analysed with the aim of identifying the time when the unexpected behaviour of the tunnel has started, its duration and pointing out its possible causes. The duration of the unexpected tunnel behaviour is identified by the means of the proposed ensemble-based change-point detection method, whose performance is compared with the results of each individual change-point method. Once the most critical change-point is identified, the works that are carried out at the tunnel site are investigated by automatically analysing the database of the work activities. However, hard copies of the spreadsheets of the works are usually used by the contractor of the works, and an electronic version is prepared only at a later date. Hence, 
some information about the works might be omitted. Other possible causes that can lead to unexpected behaviour of the tunnel, such as geophysics of the ground around the tunnel or geometry of the tunnel, are neglected due to the lack of such technical information about the tunnel. The works that are carried out at the tunnel are investigated by using the results of each change-point algorithm. Indeed, the identification of the works is achieved by looking for the activities that are carried out at the moment of the identified change-point(s). However, each individual change-point method is not able to point out clearly the most critical change-point of the tunnel behaviour, and the works that are carried out at the time of the most critical change-point cannot be identified accordingly.

In this case study, the values of the time constants $\Delta, \Phi, \Omega$, and $\Theta$, which have been introduced in Section 2.2, are optimized by expert knowledge elicitation, and they are equal to $-7 \mathrm{~h}, 15 \mathrm{~h}, 10 \mathrm{~h}$ and 0.75 , respectively.

In this paper, two particular penalty functions are considered in order to detect change-points of the monitored variable by using the individual change-point methods $i i i$ ) and $i v$ ) of Section 2.1.1:

1. a cost function, $C$, in order to detect change-points by identifying changes on the mean value of the monitored variable by applying the change-point method iii):

$$
C\left(y_{\left(\tau_{j-1}+1\right): \tau_{j}}\right)=\frac{1}{m} \sum_{j=1}^{l+1} \sum_{q=\tau_{j-1}+1}^{\tau_{j}}\left(y_{q}-\overline{y_{j}}\right)^{2}
$$

where $\bar{y}_{j}$ is the empirical mean value of the monitored behaviour of the tunnel in the segment $j, y_{q}$ is the monitored variable of the tunnel at time $q, m$ is the time duration of the monitored behaviour of the tunnel and $\tau_{j}$ is a change-point of the tunnel behaviour.

2. a cost function, $C$, which aims to identify change-points of the tunnel behaviour by pointing out changes on the slope of the system behaviour by considering an autoregressive linear model for the individual change-point method $i v$ ). The cost function is then defined as follows:

$$
C\left(y_{\left(\tau_{j-1}+1\right): \tau_{j}}\right)=\frac{3}{2} \log \left(\tau_{j}-\tau_{j-1}\right)+\frac{\tau_{j}-\tau_{j-1}}{2} \log \left(2 \pi \sigma^{*}\left(1, \tau_{j-1}+1, \tau_{j}\right)^{2}\right)
$$

where $\sigma^{*}$ is the estimation of the variance of the monitored data in the segment $\left[\tau_{j-1}, \tau_{j}\right]$, i.e. $y_{\left(\tau_{j-1}+1\right): \tau_{j}}, \tau_{j}$ is a change-point of the tunnel behaviour.

In what follows, without loss of generality, among the critical sensors of the clusters that have been identified in Section 3.3, we present two examples, in order to discuss the performance of the proposed ensemble-based change-point method with respect to the individual changepoint methods. 


\subsubsection{Change-point analysis of SAA30}

Fig. 7(a-1) shows the displacement measured by sensor SAA30_13 (i.e. the sensor number 13 of the SAA installed at $30 \mathrm{~m}$ inside the tunnel). An increase of the displacements around time 100h is recorded, as shown in Fig. 7(a-1). Fig. 7(a) depicts the performance of each individual change-point method by comparing it with the result of the proposed ensemble-based method. Each individual change-point methods is able to point out this change-point generally, as shown in Fig. 7(a-2), (a-3), (a-4) and (a-5) by the means of vertical lines and the probability of change being higher than $\Theta=0.75$ for the RuLSIF method. The change-point is identified around time $130 \mathrm{~h}$ by the RuLSIF method, which results to be at a later time than the actual initial change-point (Fig. 7(a-2)). The CUSUM, the penalty cost-function-based and the PELTbased methods are able to identify the change-point effectively around time $80 \mathrm{~h}$, as shown in Fig. 7(a-3), (a-4) and (a-5), respectively. However, each individual change-point method is not able to identify the duration of the unexpected behaviour of the tunnel, i.e. the time when the unexpected behaviour ends is not found. A second and small change-point around time 400h is identified by the CUSUM, the penalty cost-function-based and the PELT-based methods, Fig. 7(a-3), (a-4) and (a-5), and a third one at time 600h by the CUSUM method (Fig. 7(a-3)). The change-point at time $80 \mathrm{~h}$ can be caused by the works that are carried out in the first $50 \mathrm{~m}$ inside the tunnel. In a similar way, the change-point at time $130 \mathrm{~h}$, which is identified by the RuLSIF method, is due to the works in the first $50 \mathrm{~m}$ inside the tunnel. The change-point at time $400 \mathrm{~h}$ can be caused by work activities that are carried out at between 340 to $420 \mathrm{~m}$ inside of the tunnel, as reported in the database of the work activities for that time. However, the changepoint at time $400 \mathrm{~h}$ is not the most critical change-point of the tunnel behaviour, which is the one where the tunnel shows the highest change of its convergence. The most critical changepoint is not identified by each individual change-point algorithm, i.e. the start and ending time and the duration of the most critical change are not identified. Oppositely, the proposed ensemble-based change-point method is able to identify the time when the tunnel started to converge rapidly at time $74 \mathrm{~h}$. At the same time, the final time of the unexpected behaviour, i.e. when the fast convergence of the tunnel ends, is pointed out at time 161h, as shown in Fig. 7(a6). The most critical unexpected behaviour of sensor SAA30_13, and its duration, is identified by the proposed method correctly. The proposed method provides the information of the most critical behaviour of the tunnel directly, i.e. the initial time and the duration of the most critical behaviour of the tunnel are provided by the ensemble-based method in a simple and clear way. In contrast, each individual change-point method provides the information of all the changepoints of the tunnel, without assessing the duration and the criticalness of each change-point. The information of the time of occurrence of the most critical tunnel displacements is used to analyse the database of the works. Fig. 7(b) shows the works that are carried out at the tunnel site at the start and end of the fast convergence of the tunnel. It can be observed that when the track and the ballast of the rail are removed in the first $50 \mathrm{~m}$ inside the tunnel, the tunnel starts to converge rapidly. At the same time, the ground is being drained from the extra water. On the other hand, the fast convergence of the tunnel ends around 87h later, when the base stone process is started in order to fill-back the ground with new ballast. The works at the beginning of the most critical change of the tunnel behaviour can be identified correctly by using the information provided by the individual change-point algorithms. However, three out of four 
individual change-point methods do not provide information about the works that are carried out at the end of the critical behaviour of the tunnel, and the RuLSIF method provides this useful information. As a consequence, the individual change-point methods results depend on the choice of the method, and as a result, the reliability of the analysis can be jeopardized.
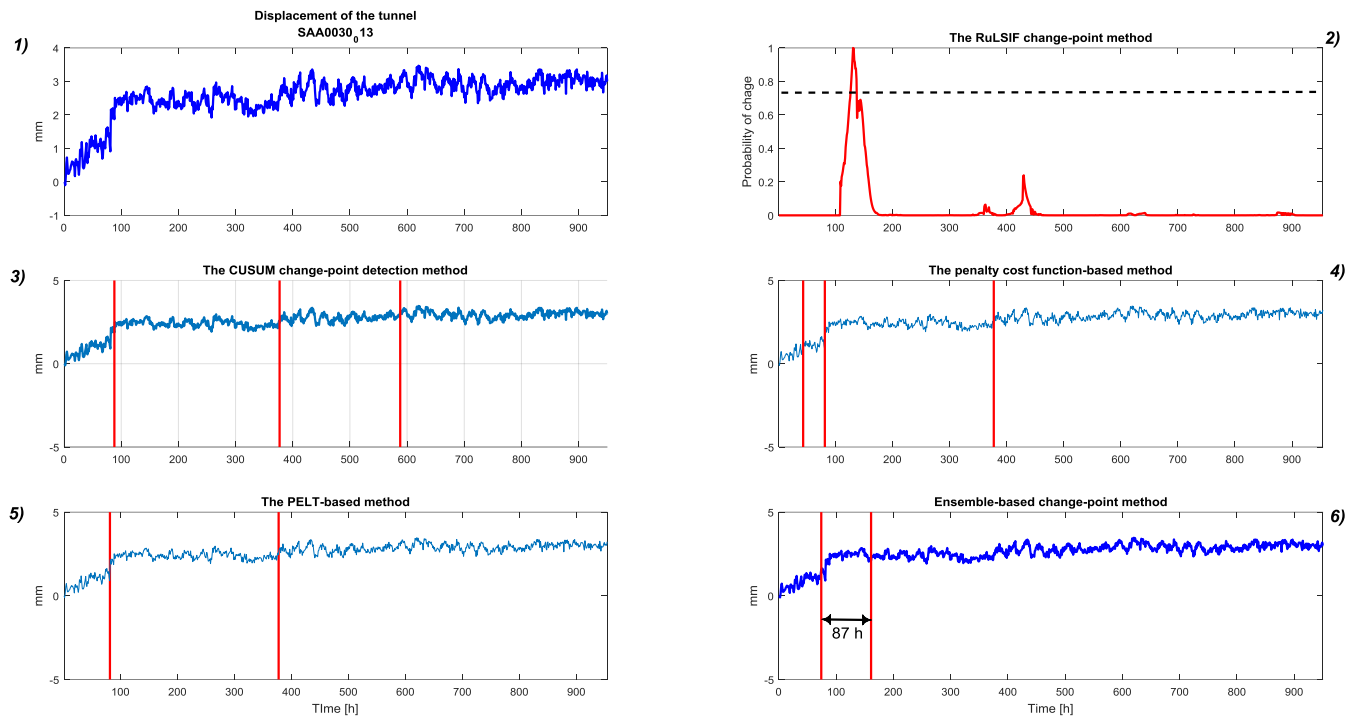

(a)

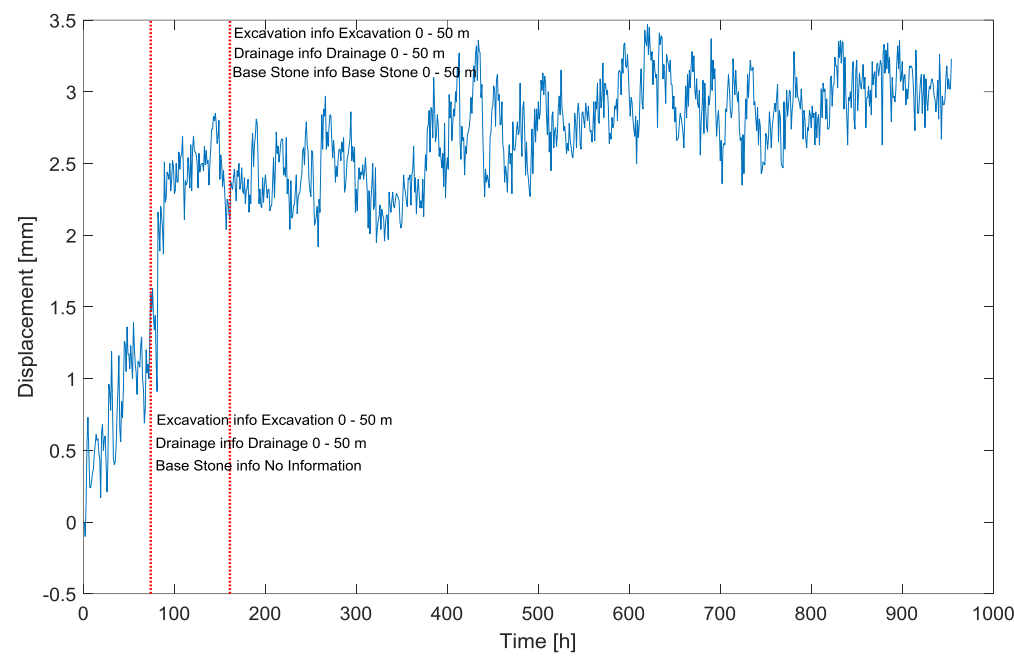

(b)

Figure 7. Change-point detection of the SAA30_13 by using the proposed ensemble-based method and each individual change-point method (a), and the corresponding work activities (b).

\subsubsection{Change-point analysis of SAA80}

An increase of the displacements of the tunnel is also measured by SAA80. The feature definition and selection process of Section 3.3 showed that the SAA80 is particularly critical, due to the fact that high values of displacement are measured all along the section of the tunnel. Indeed, almost all the sensors of the SAA80 measure an increase of the convergence of the tunnel at the beginning of the works. For example, Fig. 8(a-1) shows the displacement recorded by sensor 19 of the SAA80. Therefore, the analysis of this behaviour with the ensemble-based change-point method is needed in order to point out the time duration of this unexpected fast 
convergence of the tunnel, and the works that are carried out at the tunnel at that time. Figs. 8(a-2), (a-3), (a-4) and (a-5) show the analysis of the displacements of the tunnel by the means of each individual change-point method. Again, the RuLSIF method detects the change-point at a later time than the actual initial point of the unexpected behaviour, as shown in Fig. 8(a2 ). The CUSUM and the penalty cost-function-based agree in pointing out the first changepoint around 50h (Figs. 8(a-3) and (a-4)), whereas the PELT-based method identifies the first change-point around 80h, Fig. 8(a-5). Furthermore, the CUSUM, the penalty cost-functionbased and the PELT-based methods identifies a second change-point of the displacement of the tunnel around 100h, i.e. before that the tunnel stops to converge, as shown in Figs. 8(a-3), (a-4) and (a-5), respectively. The analysis of the database of the work activities shows that no information is available at time $50 \mathrm{~h}$, when the first change-point is identified by each individual change-point method. At time 100h, the works are carried out in the first $50 \mathrm{~m}$ inside the tunnel, and, especially, the base stone process of this section has been started. Conversely, the proposed ensemble-based change-point method identifies a change-point interval that starts at time $50 \mathrm{~h}$ and ends at time 169h (Fig. 8(a-6)). In this way, the initial point where the tunnel displacement starts to increase and the end point of the critical convergence are identified. The analysis of the database of the work activities shows that no information are available at the beginning of the critical behaviour of the tunnel. On the contrary, the tunnel stops to converge when the works are carried out at 50 to 100 meters inside the tunnel, as shown in Fig. 8(b). The base stone process of this section of the tunnel is initiated when the fast convergence of the tunnel ends, and subsequently we can conclude that when the ground is back filled with new ballast, the tunnel stops to show the unexpected fast increase of the displacement. The works identified by using the results provided by the ensemble-based method are different from those retrieved by using the results of the individual change-point algorithms. Particularly, at time 100 h, i.e. the change-point identified by the individual change-point methods, the behaviour of the tunnel is still changing rapidly, as shown in Figure 8, and the works are carried out in the first $50 \mathrm{~m}$ inside the tunnel. In contrast, the ensemble-based method points out that the fast convergence of the tunnel ends at time 160h, when the works are carried out at 50 to 100 meters inside the tunnel.

Note that similar results in terms of the performance of the proposed method, described in this section, have also been achieved during the analysis of other critical sensors, as identified in Table 1 of Section 3.3. 

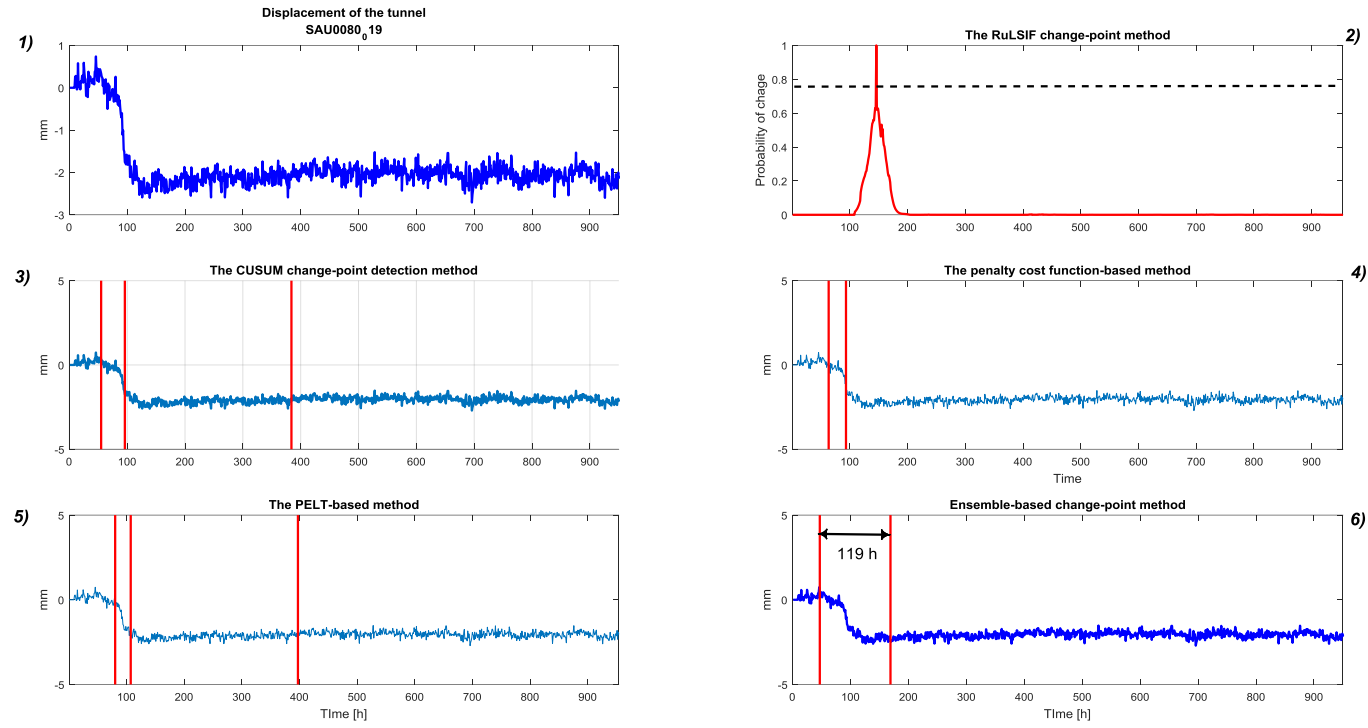

(a)

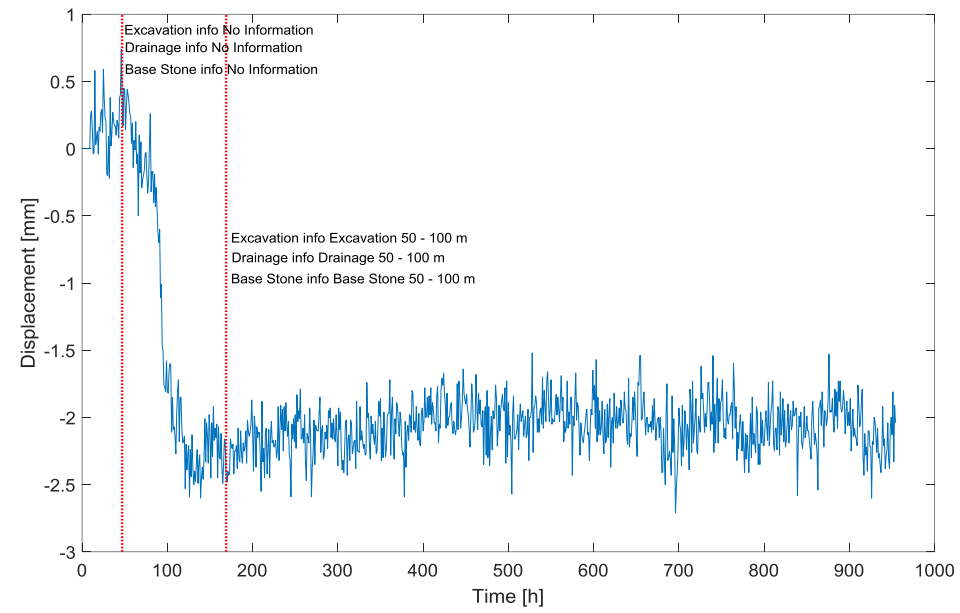

(b)

Figure 8. Change-point detection of the SAA80_19 by using the proposed ensemble-based method and each individual change-point method (a), and the corresponding work activities (b).

\subsubsection{Discussion of the results}

Table 2 shows the results of each individual change-point detection algorithm and the proposed ensemble-based method for the SAA 30, 40 and 80, i.e. the SAAs that show unexpected critical behaviour. It can be observed that the RuLSIF change-point method detects a change-point always a later time than the other change-point. This behaviour can be due to the optimization of the parameters, $\theta_{i}$, which are learned from the data, and the definition of the size of the time window, $n$. the results of the CUSUM, penalty function and PELT change-point methods agree generally in all the three SAAs analysed. The individual change-point methods provide all the change-points of the tunnel behaviour, and so the most severe change of the tunnel behaviour can be lost among all the change-points. Furthermore, individual change-point methods are unable to identify the duration of the most critical system behaviour. The works that might have caused the unexpected behaviour of the tunnel, which are identified by investigating the database of the work activities when a change-point is identified, demonstrate the usefulness 
of the proposed method. For example, the SAA30 shows the unexpected behaviour when the works are carried out in the first $50 \mathrm{~m}$ inside the tunnel. Table 2 shows that the ensemble-based method is able to point out directly the works at the initial and end time of the most critical behaviour of the tunnel, whereas the individual change-point methods are not able to correctly identify the works at the initial and end time of the critical behaviour simultaneously. Indeed, the CUSUM, the penalty function and PELT are able to correctly detect only the works at the beginning of the critical unexpected behaviour, whilst the RuLSIF method is able to correctly detect only the works at the end of the unexpected critical behaviour. For this reason, the choice of an individual change-point algorithm can threaten the reliability and robustness of the data analysis, due to different results of each individual change-point detection method. The works that are identified for the SAA40 lead to similar a conclusion. In opposition, SAA80 shows that individual change-point methods are not able to point out the works correctly. In fact, each individual change-point detects the works in the first $50 \mathrm{~m}$ inside the tunnel as a possible cause of the unexpected tunnel behaviour. However, the ensemble-based method detects that the works, which are carried out when the critical behaviour of the tunnel ends, are carried out between $50 \mathrm{~m}$ and $100 \mathrm{~m}$ inside the tunnel. This result, which is not acknowledged by the individual change-point methods, leads to conclude that a new equilibrium of the tunnel is reached due to the fact that the base stone process is initiated in the area where the SAA80 is installed.

Finally, the analysis of the three critical SAAs shows that all the critical SAAs show a common behaviour, i.e. a critical unexpected behaviour at the beginning of the works. This critical behaviour can be caused by the works that are carried out in the first $100 \mathrm{~m}$ inside the tunnel, and, as discussed in Section 3.3, on the right-hand side of the tunnel.

\begin{tabular}{|c|c|c|c|c|c|c|}
\hline & \multirow{2}{*}{$\begin{array}{l}\text { Change- } \\
\text { point } \\
\text { algorithm }\end{array}$} & \multirow{2}{*}{$\begin{array}{c}\text { Identified } \\
\text { change- } \\
\text { points [h] }\end{array}$} & \multirow{2}{*}{$\begin{array}{c}\text { Change } \\
\text { duration }[\mathrm{h}]\end{array}$} & \multicolumn{3}{|c|}{ Work activities } \\
\hline & & & & Excavation & Drainage & Base-stone \\
\hline \multirow{11}{*}{ SAA30_13 } & RuLSIF & $\begin{array}{c}\text { From } 125 \text { to } \\
167\end{array}$ & Not provided & $0-50 \mathrm{~m}$ & $0-50 \mathrm{~m}$ & $0-50 \mathrm{~m}$ \\
\hline & \multirow{3}{*}{ CUSUM } & 88 & Not provided & $0-50 \mathrm{~m}$ & $0-50 \mathrm{~m}$ & $0-50 \mathrm{~m}$ \\
\hline & & 377 & Not provided & $360-420 \mathrm{~m}$ & $360-380 \mathrm{~m}$ & $340-360 \mathrm{~m}$ \\
\hline & & 588 & Not provided & $620-680$ & $580-620 \mathrm{~m}$ & $580-620 \mathrm{~m}$ \\
\hline & \multirow{3}{*}{$\begin{array}{l}\text { Penalty } \\
\text { function }\end{array}$} & 43 & Not provided & No info & No info & No info \\
\hline & & 81 & Not provided & $0-50 \mathrm{~m}$ & $0-50 \mathrm{~m}$ & $0-50 \mathrm{~m}$ \\
\hline & & 377 & Not provided & $360-420 \mathrm{~m}$ & $360-380 \mathrm{~m}$ & $340-360 \mathrm{~m}$ \\
\hline & \multirow{2}{*}{ PELT } & 81 & Not provided & $0-50 \mathrm{~m}$ & $0-50 \mathrm{~m}$ & $0-50 \mathrm{~m}$ \\
\hline & & 377 & Not provided & $360-420 \mathrm{~m}$ & $360-380 m$ & $340-360 \mathrm{~m}$ \\
\hline & \multirow{2}{*}{ Ensemble } & 74 & \multirow{2}{*}{87} & $0-50 \mathrm{~m}$ & $0-50 \mathrm{~m}$ & $0-50 \mathrm{~m}$ \\
\hline & & 161 & & $0-50 \mathrm{~m}$ & $0-50 \mathrm{~m}$ & $0-50 \mathrm{~m}$ \\
\hline \multirow{6}{*}{ SAA40_08 } & RuLSIF & $\begin{array}{c}\text { From } 111 \text { to } \\
123\end{array}$ & Not provided & $0-50 \mathrm{~m}$ & $0-50 \mathrm{~m}$ & $0-50 \mathrm{~m}$ \\
\hline & \multirow{3}{*}{ CUSUM } & 42 & Not provided & No info & No info & No info \\
\hline & & 63 & Not provided & No info & No info & No info \\
\hline & & 625 & Not provided & No info & No info & No info \\
\hline & \multirow{2}{*}{$\begin{array}{l}\text { Penalty } \\
\text { function }\end{array}$} & 47.5 & Not provided & No info & No info & No info \\
\hline & & 58.5 & Not provided & No info & No info & No info \\
\hline
\end{tabular}




\begin{tabular}{|c|c|c|c|c|c|c|}
\hline & & 624.5 & Not provided & No info & No info & No info \\
\hline & \multirow{2}{*}{ PELT } & 33.5 & Not provided & No info & No info & No info \\
\hline & & 64.5 & Not provided & $0-50 \mathrm{~m}$ & No info & No info \\
\hline & \multirow{2}{*}{ Ensemble } & 26 & \multirow{2}{*}{105} & No info & No info & No info \\
\hline & & 131 & & $0-50 \mathrm{~m}$ & $0-50 \mathrm{~m}$ & $0-50 \mathrm{~m}$ \\
\hline \multirow{11}{*}{ SAA80_19 } & RuLSIF & $\begin{array}{c}\text { From } 152 \text { to } \\
156\end{array}$ & Not provided & $0-50 \mathrm{~m}$ & $0-50 \mathrm{~m}$ & $0-50 \mathrm{~m}$ \\
\hline & \multirow{3}{*}{ CUSUM } & 57 & Not provided & No info & No info & No info \\
\hline & & 96 & Not provided & $0-50 \mathrm{~m}$ & $0-50 \mathrm{~m}$ & $0-50 \mathrm{~m}$ \\
\hline & & 384 & Not provided & $380-420 \mathrm{~m}$ & $380-400 \mathrm{~m}$ & No info \\
\hline & \multirow{2}{*}{$\begin{array}{l}\text { Penalty } \\
\text { function }\end{array}$} & 63.5 & Not provided & $0-50 \mathrm{~m}$ & No info & No info \\
\hline & & 93.5 & Not provided & $0-50 \mathrm{~m}$ & $0-50 \mathrm{~m}$ & $0-50 \mathrm{~m}$ \\
\hline & \multirow{3}{*}{ PELT } & 80.5 & Not provided & $0-50 \mathrm{~m}$ & $0-50 \mathrm{~m}$ & No info \\
\hline & & 107.5 & Not provided & $0-50 \mathrm{~m}$ & $0-50 \mathrm{~m}$ & $0-50 \mathrm{~m}$ \\
\hline & & 397.5 & Not provided & $380-400 \mathrm{~m}$ & $420-440 \mathrm{~m}$ & No info \\
\hline & \multirow{2}{*}{ Ensemble } & 50 & \multirow{2}{*}{119} & No info & No info & No info \\
\hline & & 169 & & $50-100 \mathrm{~m}$ & $50-100 \mathrm{~m}$ & $50-100 \mathrm{~m}$ \\
\hline
\end{tabular}

Table 2. Result for each change-point detection strategy.

The proposed ensemble-based change-point method outperforms each individual change-point method, in identifying the most critical behaviour of the tunnel, by pointing out the time when the tunnel starts to converge rapidly, its duration and possible causes. Particularly, the proposed method outperforms each individual method when severe unexpected behaviour is experienced, i.e. when the displacements of the tunnel increase suddenly and rapidly. However, the proposed method is able to point out the most critical change-point of the tunnel, without providing further information regarding other smaller change-points. Decision makers can be interested in identifying and analysing the most critical behaviour of the system firstly, and once immediate actions are taken, minor changes in the system behaviour can also be analysed. In that case, a comprehensive analysis of the data can be carried out and all the vulnerabilities of the tunnel during the work activities identified. If all the change-points were to be analysed, the proposed ensemble-based change-point method can be used for this purpose by modifying its rules appropriately. For example, a decision maker can look for each change-point that has been identified by at least two individual change-point methods, rather than for the changepoint where the tunnel behaviour experiences its highest variation. Indeed, each individual change-point detection algorithm identifies different change-points, and as a consequence, a reliable and robust analysis of the most critical behaviours of the tunnel is influenced by the choice of the change-point algorithm. On the contrary, the ensemble-based method is able to identify the most critical change-point of the system behaviour, by merging the results of the different change-points methods, and thus the reliability and robustness of the identified change-point is improved. Finally, decision makers can then use the results of the ensemblebased change-point method directly, as the most critical behaviour of the tunnel is pointed out clearly, by providing the information regarding its duration and causes. In this way, the future work activities can be scheduled by guaranteeing the safety of the asset and the workforce. 


\section{Conclusion}

A large amount of data is generated by SHM systems, which monitor the health state of railway infrastructures during normal operation and maintenance or renewal activities. When maintenance or renewal activities of the infrastructures are carried out, a short-term monitoring system is usually adopted to monitor the behaviour of the infrastructure during the works. Therefore, the development of data mining techniques, which are able to analyse the large amount of measured behaviour of the infrastructure automatically, accurately and rapidly, is required in order to improve the safety of the asset and the workers. In this paper, an ensemblebased change-point detection method has been presented with the aim of identifying and diagnosing the most critical behaviours of a railway infrastructure. The proposed ensemblebased change-point detection method has been developed to overcome the limitation of the individual change-point methods. In fact, individual change-point methods can only detect abrupt changes of the behaviour of the infrastructure, without providing information regarding the severity and the duration of the identified change-points of the infrastructure behaviour. The proposed method has been verified through the analysis of a database of real-time measured displacements of a railway tunnel, which was subject to renewal works. The most critical locations of the tunnel, which required to be analysed by the means of the proposed algorithm, have been identified by using a features definition and selection process. The critical behaviour of the tunnel has been analysed by comparing the results of the proposed method with those of the individual change-point methods, with the aim of identifying when and why the unexpected behaviour of the tunnel has occurred. The results have shown that the performance of the individual change-point methods is influenced by the choice of the method, and consequently, a different assessment of the health state of the tunnel can be achieved by choosing different change-point detection methods. Conversely, it has been demonstrated that the proposed method outperformed each individual change-point detection method, by providing robust and reliable information regarding the most critical change-point of the tunnel behaviour.

Data mining techniques, such as proposed ensemble-based change-point detection method, are necessary in order to transform the large amount of data into valuable information for decision makers. The proposed method aims to provide fast and reliable information to decision makers by identifying the most critical behaviour of a tunnel. In order to provide a comprehensive analysis to the decision makers, small change-points might also need to be identified and analysed. Finally, the proposed data-mining approach can be used as an input to a prediction algorithm, such as neural networks, support vector regression, etc., in order to predict the future behaviour of the system by assessing when the unexpected behaviour will end.

\section{Acknowledgment}

Authors thank the Instrumentation and Monitoring team of AECOM Infrastructure and Environment UK Ltd., for its precious encouragement in initiating the research activity, and for the fruitful exchanges of views that have greatly improved the methodology and the manuscript. 
This project has received funding from the European Union's Horizon 2020 research and innovation programme under the Marie Skłodowska-Curie grant agreement No. 642453. 


\section{References}

[Abdoun et al., 2009] Abdoun, T., Bennett, V., Thevanayagam, S., Dobry, R., Shantz, T., Jang, D., 2009. "Wireless MEMS-based system for real-time geotechnical instrumentation of active slopes", WIT Transactions on the Built Environment, 108, pp. 617-624.

[Alves et al., 2015] Alves, V., Cury, A., Roitman, N., Magluta, C., \& Cremona, C., 2015. "Novelty detection for SHM using raw acceleration measurements", Structural Control and Health Monitoring, 22(9), 1193-1207.

[Ariznavarreta et al., 2016] Ariznavarreta-Fernández, F., González-Palacio, C., MenéndezDíaz, A., Ordoñez, C., 2016. "Measurement system with angular encoders for continuous monitoring of tunnel convergence", Tunnelling and Underground Space Technology, 56, pp. 176-185.

[Baraldi et al., 2016] Baraldi, P., Cannarile, F., Di Maio, F., Zio, E., 2016. "Hierarchical knearest neighbours classification and binary differential evolution for fault diagnostics of automotive bearings operating under variable conditions", Engineering Applications of Artificial Intelligence, 56, pp. 1-13.

[Baxter, 2015] Alan Baxter, 2015. "Network Rail A Guide to Overhead Electrification", 132787-ALB-GUN-EOH-000001, February 2015 Rev 10.

[Bhalla et al., 2005] Bhalla, S., Yang, Y.W., Zhao, J., Soh, C.K., 2005. "Structural health monitoring of underground facilities - Technological issues and challenges", Tunnelling and Underground Space Technology, 20 (5), pp. 487-500.

[Boller et al., 2015] Boller, C., Starke, P., Dobmann, G., Kuo, C.-M., Kuo, C.-H., 2015. "Approaching the assessment of ageing bridge infrastructure", Smart Structures and Systems, 15 (3), pp. 593-608.

[Brownjohn, 2007] Brownjohn, J.M.W., 2007. "Structural health monitoring of civil infrastructure", Philosophical Transactions of the Royal Society A: Mathematical, Physical and Engineering Sciences, 365 (1851), pp. 589-622.

[Chattopadhyay and Reddy, 2007] G. Chattopadhyay, V. Reddy, 2007. "Development of integrated model for assessment of operational risks in rail track", IEEE International Conference on Industrial Engineering and Engineering Management, 612 - 616.

[Carslaw et al., 2006] Carslaw, D.C., Ropkins, K., Bell, M.C., 2006. "Change-point detection of gaseous and particulate traffic-related pollutants at a roadside location", Environmental Science and Technology, 40 (22), pp. 6912-6918.

[Chen et al., 2017] Chen, T.-T., Wang, C.-H., 2017. "Fall risk assessment of bridge construction using Bayesian network transferring from fault tree analysis", Journal of Civil Engineering and Management, 23 (2), pp. 273-282.

[Di Maio et al., 2016] Di Maio, F., Vagnoli, M., Zio, E., 2016. "Transient identification by clustering based on Integrated Deterministic and Probabilistic Safety Analysis outcomes" Annals of Nuclear Energy, 87, pp. 217-227.

[Duan et al., 2006] Duan Z, Zhang K., 2006. “Data Mining Technology for Structural Health Monitoring", Pacific Science Review, 8. 
[Eskesen et al., 2004] Eskesen, S.D., Tengborg, P., Kampmann, J. and Veicherts, T.H., 2004. "Guidelines for tunnelling risk management: international tunnelling association, working group No. 2" Tunnelling and Underground Space Technology, 19(3), pp.217-237.

[Feuz et al., 2015] Feuz, K.D., Cook, D.J., Rosasco, C., Robertson, K., Schmitter-Edgecombe, M., 2015. "Automated Detection of Activity Transitions for Prompting”, IEEE Transactions on Human-Machine Systems, 45 (5), art. no. 6949090, pp. 575-585.

[Harchaoui et al., 2010] Harchaoui, Z., Lévy-Leduc, C., 2010. "Multiple change-point estimation with a total variation penalty", Journal of the American Statistical Association, 105 (492), pp. 1480-1493.

[Hong et al., 2009] Hong, E.S., Lee, I.M., Shin, H.S., Nam, S.W. and Kong, J.S., 2009. "Quantitative risk evaluation based on event tree analysis technique: application to the design of shield TBM", Tunnelling and Underground Space Technology, 24(3), pp.269-277.

[Hyun et al., 2015] Hyun, K.C., Min, S., Choi, H., Park, J. and Lee, I.M., 2015. "Risk analysis using fault-tree analysis (FTA) and analytic hierarchy process (AHP) applicable to shield TBM tunnels", Tunnelling and Underground Space Technology, 49, pp.121-129.

[Killick et al., 2012] Killick, R., Fearnhead, P., Eckley, I.A., 2012. "Optimal detection of changepoints with a linear computational cost", Journal of the American Statistical Association, 107 (500), pp. 1590-1598.

[Killick et al., 2014] Killick, R. and Eckley, I., 2014. "Changepoint: An R package for changepoint analysis", Journal of Statistical Software, 58(3), pp.1-19.

[Kilsby et al., 2017] Kilsby, P., Remenyte-Prescott, R., Andrews, J., 2017. “A modelling approach for railway overhead line equipment asset management", Reliability Engineering and System Safety, http://dx.doi.org/10.1016/j.ress.2017.02.012.

[Koepcke et al., 2016] Koepcke, L., Ashida, G., Kretzberg, J., 2016. "Single and multiple change point detection in spike trains: Comparison of different CUSUM methods", Frontiers in Systems Neuroscience, 10 (JUN), art. no. 51.

[Jain, 2010] Jain, A.K., 2010. "Data clustering: 50 years beyond K-means", Pattern Recognition Letters, 31 (8), pp. 651-666.

[IRA et al., 2015] International railway association, community of European railway and infrastructure, 2015. "Rail transport and environment, facts and figure", ISBN 976-2-74612400-4.

[Lato and Diederichs, 2014] Lato, M.J. and Diederichs, M.S., 2014. "Mapping shotcrete thickness using LiDAR and photogrammetry data: Correcting for over-calculation due to rockmass convergence", Tunnelling and Underground Space Technology, 41, pp.234-240.

[Lavielle, 2005] Lavielle, M., 2005. "Using penalized contrasts for the change-point problem", Signal Processing, 85 (8), pp. 1501-1510.

[Lee et al., 2005] Lee, J.J., Lee, J.W., Yi, J.H., Yun, C.B., Jung, H.Y., 2005. "Neural networksbased fault detection for bridges considering errors in baseline finite element models", Journal of Sound and Vibration, 280 (3-5), pp. 555-578.

[Lee et al., 2010] Lee, H.M., Kim, J.M., Sho, K., Park, H.S., 2010. “A wireless vibrating wire sensor node for continuous structural health monitoring", Smart Materials and Structures, 19 (5), art. no. 055004. 
[Li et al., 2015] Li, Y.-H., Xu, S.-D., Liu, J.-P., 2015. "A new convergence monitoring system for tunnel or drift based on draw-wire displacement sensors", Tunnelling and Underground Space Technology, 49, pp. 92-97.

[Li et al., 2016] Li, H. N., Ren, L., Jia, Z. G., Yi, T. H., \& Li, D. S., 2016. "State-of-the-art in structural health monitoring of large and complex civil infrastructures", Journal of Civil Structural Health Monitoring, 6(1), 3-16.

[Lipowsky et al., 2010] Lipowsky, H., Staudacher, S., Bauer, M., Schmidt, K.-J., 2010. "Application of bayesian forecasting to change detection and prognosis of gas turbine performance", Journal of Engineering for Gas Turbines and Power, 132 (3), art. no. 031602.

[Liu et al., 2013] Liu, S., Yamada, M., Collier, N., Sugiyama, M., 2013. "Change-point detection in time-series data by relative density-ratio estimation", Neural Networks, 43, pp. 72-83.

[Maleki et al., 2016] Maleki, S., Bingham, C., Zhang, Y., 2016. "Development and Realization of Changepoint Analysis for the Detection of Emerging Faults on Industrial Systems", IEEE Transactions on Industrial Informatics, 12 (3), art. no. 7458838, pp. 1180-1187.

[Misiunas et al., 2006] Misiunas, D., Vitkovský, J., Olsson, G., Lambert, M., Simpson, A., 2006. "Failure monitoring in water distribution networks", Water Science and Technology, 53 (4-5), pp. 503-511.

[Miura et al., 2005] Miura, S., Yamamoto, T., Kuronuma, I. and Imai, M., 2005. "Vision metrology applied for configuration and displacement", International Journal of the JCRM, 1(1), pp.1-6.

[Mohamad et al., 2012] Mohamad, H., Soga, K., Bennett, P.J., Mair, R.J., Lim, C.S., 2012. "Monitoring twin tunnel interaction using distributed optical fiber strain measurements", Journal of Geotechnical and Geoenvironmental Engineering, 138 (8), pp. 957-967.

[Montes De Oca et al., 2010] Montes De Oca, V., Jeske, D.R., Zhang, Q., Rendon, C., Marvasti, M., 2010. "A cusum change-point detection algorithm for non-stationary sequences with application to data network surveillance", Journal of Systems and Software, 83 (7), pp. 12881297.

[Ordoñez et al., 2016] Ariznavarreta-Fernández, F., González-Palacio, C., Menéndez-Díaz, A., Ordoñez, C., 2016. "Measurement system with angular encoders for continuous monitoring of tunnel convergence”, Tunnelling and Underground Space Technology, 56, pp. 176-185.

[Rajabi et al., 2017] Rajabi, M., Rahmannejad, R., Rezaei, M., Ganjalipour, K., 2017. "Evaluation of the maximum horizontal displacement around the power station caverns using artificial neural network", Tunnelling and Underground Space Technology, 64, pp. 51-60.

[Reeves et al., 2007] Reeves, J., Chen, J., Wang, X. L., Lund, R., and Lu., Q., 2007. "A review and comparison of changepoint detection techniques for climate data", Journal of Applied Meteorology and Climatology, 46(6):900-915.

[Rezaie et al., 2007] Rezaie, K., Amalnik, M.S., Gereie, A., Ostadi, B. and Shakhseniaee, M., 2007. "Using extended Monte Carlo simulation method for the improvement of risk management: Consideration of relationships between uncertainties", Applied Mathematics and Computation, 190(2), pp.1492-1501.

[Rousseeuw, 1987] Rousseeuw, P.J., 1987. "Silhouettes: A graphical aid to the interpretation and validation of cluster analysis", Journal of Computational and Applied Mathematics, 20 (C), pp. 53-65. 
[Simeoni and Zanei, 2009] Simeoni, L. and Zanei, L., 2009. "A method for estimating the accuracy of tunnel convergence measurements using tape distometers", International Journal of Rock Mechanics and Mining Sciences, 46(4), pp.796-802.

[Sousa and Einstein, 2012] Sousa, R.L. and Einstein, H.H., 2012. "Risk analysis during tunnel construction using Bayesian Networks: Porto Metro case study", Tunnelling and Underground Space Technology, 27(1), pp.86-100.

[Tartakovsky et al., 2014] Tartakovsky, A., Basseville, M., and. Nikiforov, I.V, 2014. "Sequential Analysis: Hypothesis Testing and Changepoint Detection", Taylor \& Francis, ISBN 9781439838204.

[Tickle et al., 2010] Tickle, A.J., Harvey, P.K., Smith, J.S., 2010. "Applications of a Morphological Scene Change Detection (MSCD) for visual leak and failure identification in process and chemical engineering", Proceedings of SPIE - The International Society for Optical Engineering, 7833, art. no. 78330W.

[Wang et al., 2017] Wang, B., Zhang, Z., He, C., Zheng, H., 2017. "Implementation of a longterm monitoring approach for the operational safety of highway tunnel structures in a severely seismic area of China", Structural Control Health Monitoring, https://doi.org/10.1002/stc.1993.

[Yazdani-Chamzini, 2014] Yazdani-Chamzini, A., 2014. "Proposing a new methodology based on fuzzy logic for tunnelling risk assessment", Journal of Civil Engineering and Management, 20 (1), pp. 82-94.

[Zhou et al., 2015] Zhou, F., Zhang, W., Sun, K., \& Shi, B., 2015. "Health state evaluation of shield tunnel SHM using fuzzy cluster method", Nondestructive Evaluation and Health Monitoring pp. 94371 J. 